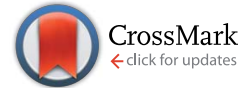

Cite this: RSC Adv., 2016, 6, 47023
Received 13th March 2016 Accepted 3rd May 2016 DOI: $10.1039 / c 6 r a 06671 b$ www.rsc.org/advances

\section{Uncatalyzed reactions of 4,4'-diphenylmethane- diisocyanate with polymer polyols as revealed by matrix-assisted laser desorption/ionization mass spectrometry $\dagger$}

Tibor Nagy, ${ }^{a}$ Borbála Antal, ${ }^{a}$ Anita Dékány-Adamoczky, ${ }^{a}$ József Karger-Kocsis, ${ }^{b}$ Miklós Zsuga ${ }^{a}$ and Sándor Kéki ${ }^{\star a}$

The uncatalyzed reactions of polyols including polypropylene glycol (PPG, $M_{n}=2000 \mathrm{~g} \mathrm{~mol}^{-1}$ ), polytetrahydrofuran (PTHF, $M_{n}=1000 \mathrm{~g} \mathrm{~mol}^{-1}$ ), poly $\left(\varepsilon\right.$-caprolactone)-diol (PCLD, $M_{n}=2000 \mathrm{~g} \mathrm{~mol}^{-1}$ ) and polypropylene glycol glycerol triether (PPG_GL, $M_{n}=1000 \mathrm{~g} \mathrm{~mol}^{-1}$ ) with 4,4'-diphenylmethanediisocyanate (MDI) were studied using matrix-assisted laser desorption/ionization time-of-flight mass spectrometry (MALDI-TOF MS). The reactions between these polyols and MDI were monitored in time and first-order consecutive reaction kinetics for the formations of polyols end-capped with MDI units were established. The fractional MALDI-TOF MS intensities obtained for the different polymer series at various reaction times were converted into molar fractions versus time data by means of the estimated relative MALDI-TOF MS response factors from which the corresponding rate constants and the relative reactivities of the hydroxyl groups of polyols were determined. It was found that the pseudo first-order rate constants for the polyol-MDI reaction decreased in the order of PCLD $>$ PTHF $>$ PPG $\approx$ PPG_GL. It was also ascertained that the reactivity of the unreacted hydroxyl groups of the diols does not change significantly after the first one has reacted. On the contrary, in the case of PPG_GL it was found that the reactions of the hydroxyl groups with MDI proceed faster after any of the three hydroxyl groups has reacted, suggesting a positive substitution effect for this system.

\section{Introduction}

Polyurethanes (PUs) are one of the most versatile classes of industrial polymers whose applications cover various important areas ranging from coatings through elastomers to different types of biomaterials. ${ }^{1-3}$ PUs have recently received considerable attention as shape-memory materials. ${ }^{4-6}$ The production routes to PUs are based on the addition reactions of isocyanates with functionalities of two or higher with different types of polyols. Proper selection of the isocyanate and the polyol components allows one to tailor the properties of the resulting PUs for various applications. Evidently, the reactions of the isocyanate group with the hydroxyl groups of polyols play an essential role in the synthesis of PUs. Thus, due to the practical importance of PUs, the catalyzed and uncatalyzed reactions of different isocyanates with alcohols under various experimental conditions have been extensively studied from the early ages of the

${ }^{a}$ Department of Applied Chemistry, University of Debrecen, Egyetem tér1, H-4032 Debrecen, Hungary. E-mail: keki.sandor@science.unideb.hu

${ }^{b}$ Department of Polymer Engineering, Budapest University of Technology and Economics, Müegyetem rkp. 3, H-1111 Budapest, Hungary

$\dagger$ Electronic supplementary information (ESI) available. See DOI: $10.1039 / \mathrm{c} 6 \mathrm{ra06671b}$ polyurethane industry. ${ }^{7-10}$ The kinetic studies on this topic have primarily focused on the reactions of mono- and industrially important diisocyanates with monofunctional alcohols to obtain the reaction order for both the isocyanate and alcohol components and to establish the reaction mechanism. ${ }^{11-20}$ The kinetics of the reaction between diisocyanates and polyols and polymer polyols with functionalities of two or higher are also well-documented.$^{21-37}$ In most of these investigations the reaction was monitored using, e.g., titration method, ${ }^{21,22}$ FT-IR spectroscopy, ${ }^{23-30}$ rheological measurements ${ }^{29,31}$ and sizeexclusion chromatography (SEC)..$^{32}$ Moreover, when a diol has been reacted with isocyanate the reaction mixture at an intermediate reaction time will surely contain the unreacted diols, the diols with one reacted and diols with two reacted $\mathrm{OH}$ groups. Titration and spectroscopic methods such as FT-IR and UV spectroscopy are, in most cases, capable of determining only the fraction of the number of reacted isocyanate and/or $\mathrm{OH}$ groups. Thus, the above mentioned methods may not provide information on the distribution of the various species present in the reaction mixture. In addition, as the molecular weight of the polyol component is increased the concentration of the "spectroscopically active" groups is thereby reduced making both the qualitative and quantitative interpretations very 
difficult. On the contrary, due to the well-resolved peaks, mass spectrometric methods, and especially those based on softionization such as matrix-assisted laser desorption/ionization mass spectrometry (MALDI MS) ${ }^{38}$ and electrospray ionization (ESI-MS) ${ }^{39}$ are capable of detecting various species (including those which have high molecular weights) present in the reaction mixture.

In this article, we report a detailed MALDI-TOF MS investigations on the reactions of 4,4'-diphenylmethane-diisocyanate (MDI) with polymer polyols including polypropylene glycol (PPG), polytetrahydrofuran (PTHF), polycaprolactone diol (PCLD) and a triol polypropylene glycol glycerol triether (PPG_GL, Rokopol G1000). The goals of this study were to gain information on the distributions of the intermediates and products formed during the reaction and to determine the reactivities of the hydroxyl groups of polymeric polyols. To the best of our knowledge such kind of investigation has not been reported yet.

\section{Experimental}

\section{Chemicals}

4,4'-Diphenylmethane-diisocyanate (MDI), polycaprolactonediol (PCLD, $M_{\mathrm{n}}=2000 \mathrm{~g} \mathrm{~mol}^{-1}$ ), toluene and tetrahydrofuran were received from Sigma-Aldrich (Taufkirchen, Germany). Polypropylene glycol (PPG, $M_{\mathrm{n}}=2000 \mathrm{~g} \mathrm{~mol}^{-1}$ ) and polypropylene glycol glycerol triether (PPG_GL, $M_{\mathrm{n}}=1000 \mathrm{~g} \mathrm{~mol}^{-1}$, Rokopol G1000) were obtained from BorsodChem (Kazincbarcika, Hungary) and PCC Rokita SA (Brzeg Dolny, Poland), respectively. Methanol and tetrahydrofurane were purchased from VWR International (Leuven, Belgium), respectively, and were used without further purification. Toluene was purified and dried according to the well-know procedure. ${ }^{40}$ Polymer polyols were dried at $50{ }^{\circ} \mathrm{C}$ in a vacuum oven for 48 hours.

\section{General procedure for performing the reactions of diisocyanates with alcohols}

Into a flask of $5 \mathrm{~mL}$, dry toluene $(3 \mathrm{~mL}$ ) and calculated amount of MDI were introduced under nitrogen atmosphere. The flask was thermostated at $80^{\circ} \mathrm{C}$. The reactions were started by addition of the calculated amount of the corresponding polymer polyols (PCLD or PPG or PTHF or PPG_GL) dissolved in toluene of $1 \mathrm{~mL}$. The reaction mixture was further diluted with toluene to obtain a reaction volume of $5 \mathrm{~mL}$. The initial concentrations of MDI and the polymer polyols were $0.32 \mathrm{M}$ and $0.01 \mathrm{M}$, respectively. For the MALDI-TOF MS investigations, after predetermined time intervals, samples of $100 \mu \mathrm{L}$ were taken out from the reaction mixture and added to a mixture of methanol/ tetrahydrofuran $(2: 1 \mathrm{v} / \mathrm{v})$ to quench the unreacted isocyanate groups.

\section{Determination of the primary and secondary hydroxyl group content of PPG based samples}

The primary and secondary group contents of PPG and PPG_GL samples were determined similarly to the method published in the literature. ${ }^{\mathbf{4 1 , 4 2}}$ Samples of $50-100 \mathrm{mg}$ were dissolved in
$\mathrm{CDCl}_{3}$ of $500 \mu \mathrm{L}$ to which trifluoroacetic anhydride in a 10-fold molar excess was added. After 3 hours reaction time the solutions were analyzed by ${ }^{1} \mathrm{H}-\mathrm{NMR}$. The primary and the secondary group contents were calculated using the integrals of proton signals at $4.2 \mathrm{ppm}$, characteristics of $-\mathrm{CH}\left(\mathrm{CH}_{3}\right)-\mathrm{CH}_{2}-\mathrm{OCOCF}_{3}$ and, at $5.2 \mathrm{ppm}$ originating from $-\mathrm{CH}_{2}-\mathrm{CH}\left(\mathrm{CH}_{3}\right) \mathrm{OCOCF}_{3}$.

\section{Matrix-assisted laser desorption/ionization time-of-flight mass spectrometry (MALDI-TOF MS)}

The MALDI-TOF MS measurements were performed with a Bruker BIFLEX III ${ }^{\mathrm{TM}}$ mass spectrometer equipped with a timeof-flight (TOF) mass analyzer. In all cases $19 \mathrm{kV}$ acceleration voltage was used with pulsed ion extraction (PIETM). The positive ions were detected in the reflectron mode $(20 \mathrm{kV})$. A nitrogen laser ( $337 \mathrm{~nm}, 3 \mathrm{~ns}$ pulse width, $10^{6}$ to $10^{7} \mathrm{~W} \mathrm{~cm}^{-2}$ ) operating at $4 \mathrm{~Hz}$ was used to produce laser desorption and 300 shots were summed. The MALDI-TOF MS spectra were externally calibrated with poly(ethylene glycol)s $\left(M_{\mathrm{n}}=1450 \mathrm{~g} \mathrm{~mol}^{-1}\right.$ and $\left.M_{\mathrm{n}}=4000 \mathrm{~g} \mathrm{~mol}^{-1}\right)$.

Samples for MALDI-TOF MS were prepared with 2,5-dihydroxy benzoic acid (DHB) matrix dissolved in tetrahydrofuran at a concentration of $20 \mathrm{mg} \mathrm{mL}{ }^{-1}$. Analyte samples were obtained by mixing $100 \mu \mathrm{L}$ reaction mixture with methanol $(400 \mu \mathrm{L})$ and tetrahydrofuran $(200 \mu \mathrm{L})$ and sodium trifluoroacetate dissolved in tetrahydrofuran at a concentration of $5 \mathrm{mg} \mathrm{mL}^{-1}$ (used as the cationization agent to promote ionization). The solutions were mixed in a $10: 2: 1(\mathrm{v} / \mathrm{v})$ ratio (matrix/analyte/cationization agent). A volume of $0.5 \mu \mathrm{L}$ of the solution was deposited onto a metal sample plate and allowed to air-dry.

\section{Electrospray quadrupole time-of-flight mass spectrometry (ESI-QTOF MS)}

The MS and MS/MS measurements were performed with a MicroTOF-Q type Qq-TOF MS instrument (Bruker Daltonik, Bremen, Germany) using an ESI source with positive ion mode. The sample solutions were introduced directly into the ESI source with a syringe pump (Cole-Parmer Ins. Co., Vernon Hills, IL, USA) at a flow rate of $3 \mu \mathrm{L} \mathrm{min}{ }^{-1}$. The spray voltage was set to $4 \mathrm{kV}$. The temperature of the drying gas $\left(\mathrm{N}_{2}\right)$ was kept at $180{ }^{\circ} \mathrm{C}$. For the tandem MS measurements nitrogen was used as the collision gas and collision energies of $142 \mathrm{eV}$ and $178 \mathrm{eV}$ were applied (in the laboratory frame). The precursor ions for MS/MS were selected with an isolation width of $4 \mathrm{~m} / \mathrm{z}$ units. The MS and MS/MS spectra were accumulated and recorded by means of a digitizer at a sampling rate of $2 \mathrm{GHz}$. The mass spectra were calibrated externally using the exact masses of the clusters generated from the electrosprayed solution of sodium trifluoroacetate (NaTFA). The recorded mass spectra were evaluated with the DataAnalysis 3.4 software from Bruker. Samples for the ESI-MS and ESI-MS/MS measurements were prepared using $10 \mu \mathrm{L}$ of methanol-quenched reaction mixtures obtained at different reaction times and diluted with methanol to 1000 $\mu \mathrm{L}$. 


\section{Nuclear magnetic resonance spectroscopy (NMR)}

${ }^{1} \mathrm{H}$-NMR spectra were recorded in $\mathrm{CDCl}_{3}$ at $25{ }^{\circ} \mathrm{C}$ on a Bruker AM 360 spectrometer at $360 \mathrm{MHz}$ with tetramethylsilane as the internal standard.

\section{Evaluation of the kinetic measurements}

The reaction steps of MDI with diols including PPG, PTHF and PCLD together with the products obtained after quenching the reaction mixture with methanol are depicted in Scheme 1.

The MALDI-TOF MS intensities belonging to the corresponding oligomer series obtained on the quenched reaction mixture were summed and these intensities were used to calculate the fractional intensities $\left(F_{\mathrm{A}_{n}}, F_{\mathrm{B}_{n}}\right.$ and $\left.F_{\mathrm{C}_{n}}\right)$ for series $\mathrm{A}_{n}$, $\mathrm{B}_{n}$ and $\mathrm{C}_{n}$ as given by eqn (1)-(3).

$$
\begin{gathered}
F_{\mathrm{A}_{n}}=\frac{I_{\mathrm{A}_{n}}}{I_{\mathrm{A}_{n}}+I_{\mathrm{B}_{n}}+I_{\mathrm{C}_{n}}} \\
F_{\mathrm{B}_{n}}=\frac{I_{\mathrm{B}_{n}}}{I_{\mathrm{A}_{n}}+I_{\mathrm{B}_{n}}+I_{\mathrm{C}_{n}}} \\
F_{\mathrm{C}_{n}}=1-F_{\mathrm{A}_{n}}-F_{\mathrm{B}_{n}}
\end{gathered}
$$

where $I_{\mathrm{A}}, I_{\mathrm{B}}$ and $I_{\mathrm{C}}$ are the summed MALDI-TOF MS intensities for the oligomer series $\mathrm{A}_{n}, \mathrm{~B}_{n}$ and $\mathrm{C}_{n}$, respectively ( $c f$. Scheme 1b).

Assuming linear relationship between the MALDI-TOF MS intensities and the concentrations of the corresponding oligomer series eqn (4)-(6) become as:

$$
I_{\mathrm{A}_{n}}=f_{\mathrm{A}}\left[\mathrm{A}_{n}\right]
$$

$$
\begin{gathered}
I_{\mathrm{B}_{n}}=f_{\mathrm{B}}\left[\mathrm{B}_{n}\right] \\
I_{\mathrm{C}_{n}}=f_{\mathrm{C}}\left[\mathrm{C}_{n}\right]
\end{gathered}
$$

where $f_{\mathrm{A}}, f_{\mathrm{B}}$ and $f_{\mathrm{C}}$ are the MALDI-TOF MS response factors for series $\mathrm{A}_{n}, \mathrm{~B}_{n}$ and $\mathrm{C}_{n}$, respectively.

Using eqn (4)-(6), the molar fractions, i.e., $X_{\mathrm{A}_{n}}, X_{\mathrm{B}_{n}}$ and $X_{\mathrm{C}_{n}}$ for series $\mathrm{A}_{n}, \mathrm{~B}_{n}$ and $\mathrm{C}_{n}$ can be given by eqn (7)-(9) as:

$$
\begin{gathered}
X_{\mathrm{A}_{n}}=\frac{I_{\mathrm{A}_{n}}}{I_{\mathrm{A}_{n}}+\left(f_{\mathrm{A}} / f_{\mathrm{B}}\right) I_{\mathrm{B}_{n}}+\left(f_{\mathrm{A}} / f_{\mathrm{C}}\right) I_{\mathrm{C}_{n}}} \\
X_{\mathrm{B}_{n}}=\frac{\left(f_{\mathrm{A}} / f_{\mathrm{B}}\right) I_{\mathrm{B}_{n}}}{I_{\mathrm{A}_{n}}+\left(f_{\mathrm{A}} / f_{\mathrm{B}}\right) I_{\mathrm{B}_{n}}+\left(f_{\mathrm{A}} / f_{\mathrm{C}}\right) I_{\mathrm{C}_{n}}} \\
X_{\mathrm{C}_{n}}=1-X_{\mathrm{A}_{n}}-X_{\mathrm{B}_{n}}
\end{gathered}
$$

According to the reactions presented in Scheme 1: (i) assuming pseudo-first order rate dependence for the diols, and (ii) taking into account that each of the isocyanate groups and hydroxyl groups have the same reactivity due to symmetrical nature of the MDI and diols, the variation of the corresponding fraction of the oligomer series with time can be described by eqn (10)-(12)

$$
\begin{gathered}
X_{\mathrm{A}_{n}}(t)=\mathrm{e}^{-4 k_{1} t} \\
X_{\mathrm{B}_{n}}(t)=\frac{4 k_{1}}{2 k_{2}-4 k_{1}}\left(\mathrm{e}^{-4 k_{1} t}-\mathrm{e}^{-2 k_{2} t}\right) \\
X_{\mathrm{C}_{n}}(t)=1-X_{\mathrm{A}_{n}}(t)-X_{\mathrm{B}_{n}}(t)
\end{gathered}
$$

where $k_{1}$ and $k_{2}$ are the pseudo first-order rate constants.

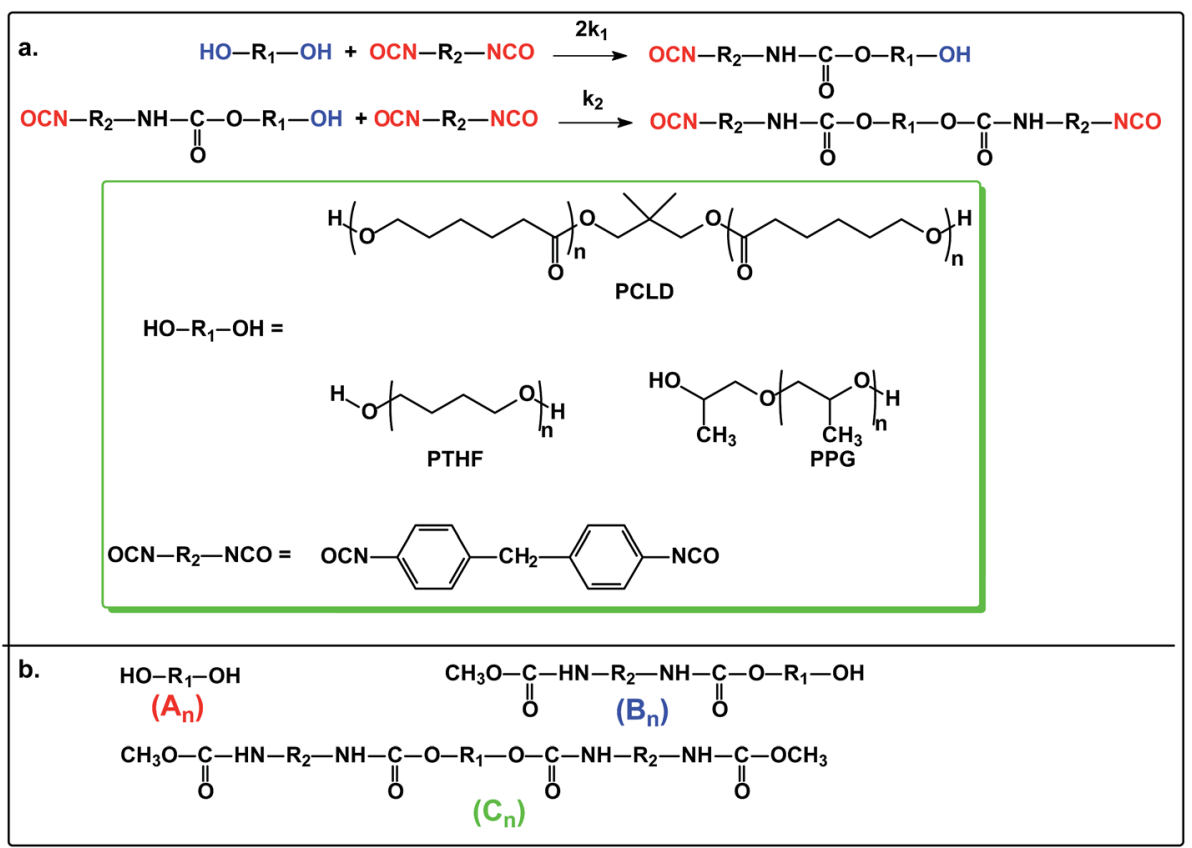

Scheme 1 The reactions of polymer diols (PPG, PTHF and PCLD) with MDI (a) and the products obtained after quenching the reaction mixture by methanol (b). 
The reaction steps of MDI with a triol, i.e., polypropylene glycol glycerol triether (PPG_GL, Rokopol G1000) and the products obtained after quenching the reaction mixture with methanol are shown in Scheme 2.

Similarly to the MDI-polymer diols system, the MALDI-TOF MS intensities belonging to the corresponding oligomer series obtained on the quenched reaction mixture were summed to calculate the fractional intensities for series $\mathrm{A}_{n}, \mathrm{~B}_{n}, \mathrm{C}_{n}$ and $\mathrm{D}_{n}$. Assuming linear relationships between the MALDI-TOF MS intensities and the concentrations of corresponding oligomer series, the molar fractions, i.e., $X_{\mathrm{A}_{n}}, X_{\mathrm{B}_{n}}, X_{\mathrm{C}_{n}}$ and $X_{\mathrm{D}_{n}}$ for series $\mathrm{A}_{n}$, $\mathrm{B}_{n}, \mathrm{C}_{n}$ and $\mathrm{D}_{n}$ ( $c f$. Scheme $2 \mathrm{~b}$ ), respectively, can be given as:

$$
\begin{gathered}
X_{\mathrm{A}_{n}}=\frac{I_{\mathrm{A}_{n}}}{I_{\mathrm{A}_{n}}+\left(f_{\mathrm{A}} / f_{\mathrm{B}}\right) I_{\mathrm{B}_{n}}+\left(f_{\mathrm{A}} / f_{\mathrm{C}}\right) I_{\mathrm{C}_{n}}+\left(f_{\mathrm{A}} / f_{\mathrm{D}}\right) I_{\mathrm{D}_{n}}} \\
X_{\mathrm{B}_{n}}=\frac{\left(f_{\mathrm{A}} / f_{\mathrm{B}}\right) I_{\mathrm{B}_{n}}}{X_{\mathrm{A}_{n}}+\left(f_{\mathrm{A}} / f_{\mathrm{B}}\right) I_{\mathrm{B}_{n}}+\left(f_{\mathrm{A}} / f_{\mathrm{C}}\right) I_{\mathrm{C}_{n}}+\left(f_{\mathrm{A}} / f_{\mathrm{D}}\right) I_{\mathrm{D}_{n}}}=\frac{\left(f_{\mathrm{A}} / f_{\mathrm{C}}\right) I_{\mathrm{C}_{n}}}{I_{\mathrm{A}_{n}}+\left(f_{\mathrm{A}} / f_{\mathrm{B}}\right) I_{\mathrm{B}_{n}}+\left(f_{\mathrm{A}} / f_{\mathrm{C}}\right) I_{\mathrm{C}_{n}}+\left(f_{\mathrm{A}} / f_{\mathrm{D}}\right) I_{\mathrm{D}_{n}}} \\
X_{\mathrm{D}_{n}}=1-X_{\mathrm{A}_{n}}-X_{\mathrm{B}_{n}}-X_{\mathrm{C}_{n}}
\end{gathered}
$$

where $f_{\mathrm{A}}, f_{\mathrm{B}}, f_{\mathrm{C}}$ and $f_{\mathrm{D}}$ are the MALDI-TOF MS response factors for series $\mathrm{A}_{n}, \mathrm{~B}_{n} \mathrm{C}_{n}$, and $\mathrm{D}_{n}$, respectively.

Assuming pseudo-first order rate dependence for the polymer triol and equal reactivities for each of the isocyanate and hydroxyl groups, the change of the corresponding fraction of the oligomer series in time are given by eqn (17)-(20).

$$
\begin{gathered}
X_{\mathrm{A}_{n}}(t)=\mathrm{e}^{-6 k_{1} t} \\
X_{\mathrm{B}_{n}}(t)=\frac{6 k_{1}}{4 k_{2}-6 k_{1}}\left(\mathrm{e}^{-6 k_{1} t}-\mathrm{e}^{-4 k_{2} t}\right)
\end{gathered}
$$

$$
\begin{aligned}
X_{\mathrm{C}_{n}}(t)= & \frac{24 k_{1} k_{2}}{\left(6 k_{1}-4 k_{2}\right)\left(6 k_{1}-2 k_{3}\right)}\left(\mathrm{e}^{-6 k_{1} t}-\mathrm{e}^{-2 k_{3} t}\right) \\
& +\frac{24 k_{1} k_{2}}{\left(6 k_{1}-4 k_{2}\right)\left(4 k_{2}-2 k_{3}\right)}\left(\mathrm{e}^{-2 k_{3} t}-\mathrm{e}^{-4 k_{2} t}\right) \\
X_{\mathrm{D}_{n}}(t) & =1-X_{\mathrm{A}_{n}}(t)-X_{\mathrm{B}_{n}}(t)-X_{\mathrm{C}_{n}}(t)
\end{aligned}
$$

where $k_{1}, k_{2}$ and $k_{3}$ are the pseudo first-order rate constants.

For fitting of the parameters of eqn (10)-(12) and (17)-(20) to the experimental kinetic data and thus to determine the corresponding rate constants a home-made parameter estimation software based the Gauss-Newton-Marquardt procedure was applied. ${ }^{43}$

\section{Estimation of the relative MALDI-TOF MS response factors}

Since no internal standards for the oligomer series are available either for the MDI-polymer-diol or for the MDI-polymer-triol reactions we have developed a method to estimate the relative MALDI-TOF MS response factors of the oligomer series with respect to series $\mathrm{A}_{n}$. At different reaction times, i.e., at various product distributions, samples were taken out from the reaction mixtures and quenched with methanol similarly as described before. To each quenched samples, polyethylene glycol (PEG, $M_{\mathrm{n}}$ $\left.=1450 \mathrm{~g} \mathrm{~mol}^{-1}\right)$ or polypropylene glycol $\left(M_{\mathrm{n}}=1000 \mathrm{~g} \mathrm{~mol}^{-1}\right)$ were added as internal standard (reference). The intensities of the reference were compared to those of the different series, and the corresponding relative response factors were estimated by means of eqn (S1) and (S2) (ESI†) using multi regression analysis.

\section{Results and discussion}

\section{Reaction of polymer diols with MDI}

Polymer diols including polypropylene glycol (PPG, $M_{\mathrm{n}}=2000 \mathrm{~g}$ $\left.\mathrm{mol}^{-1}\right)$, polytetrahydrofuran $\left(M_{\mathrm{n}}=1000 \mathrm{~g} \mathrm{~mol} \mathrm{~mol}^{-1}\right)$ and

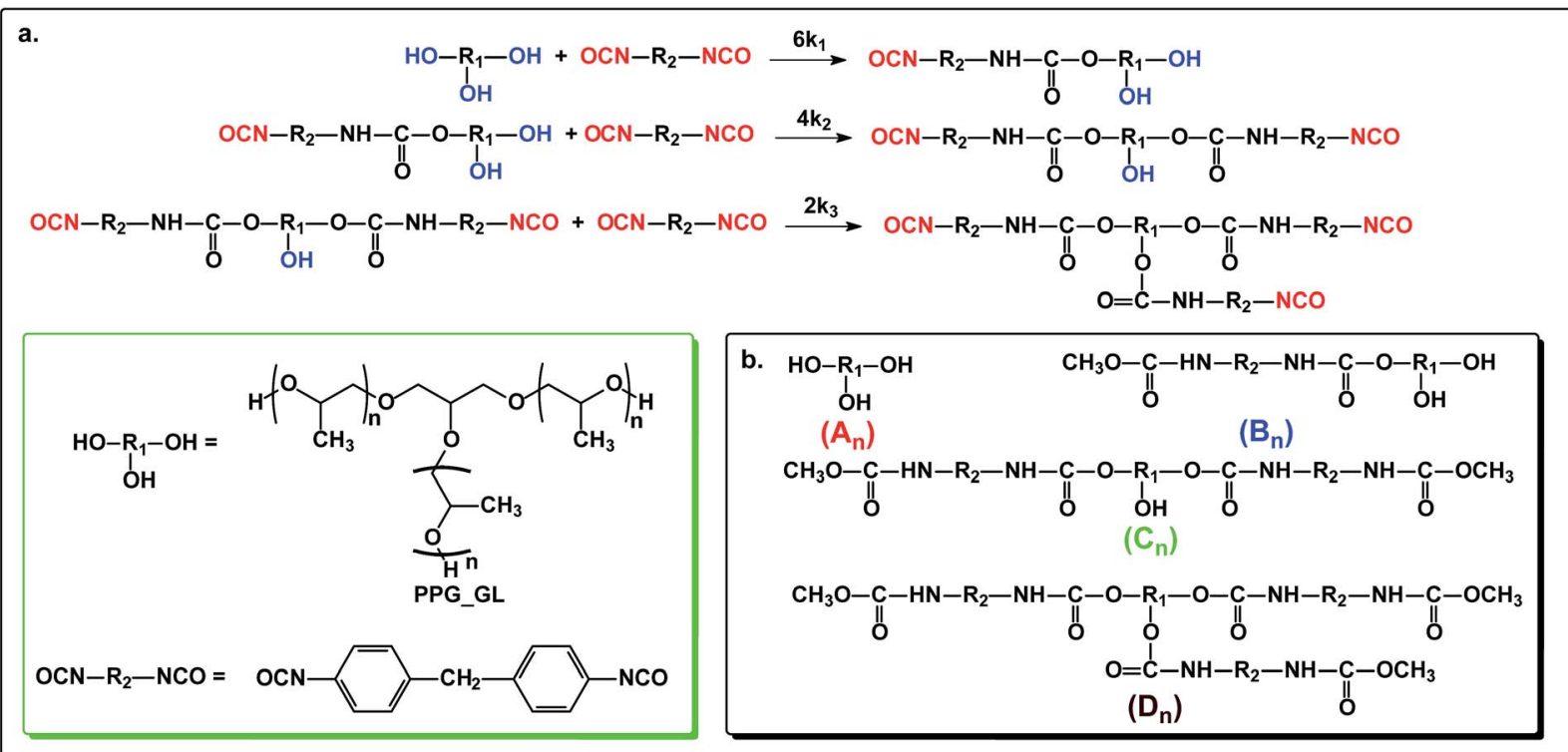

Scheme 2 The reaction of polypropylene glycol glycerol triether (PPG_GL) with MDI (a) and the products obtained after quenching the reaction mixture by methanol (b). 
polycaprolactone diol (PCLD, $M_{\mathrm{n}}=2000 \mathrm{~g} \mathrm{~mol}^{-1}$ ) were reacted with MDI in high molar excess to polymer diols in toluene at 80 ${ }^{\circ} \mathrm{C}$. After predetermined time intervals, depending on the rates of reactions, samples were taken out of the reaction mixture, quenched with methanol and analyzed by MALDI-TOF MS. The MALDI-TOF MS spectra of the methanol-quenched reaction mixture obtained in the reaction of MDI with PPG are shown in Fig. 1.

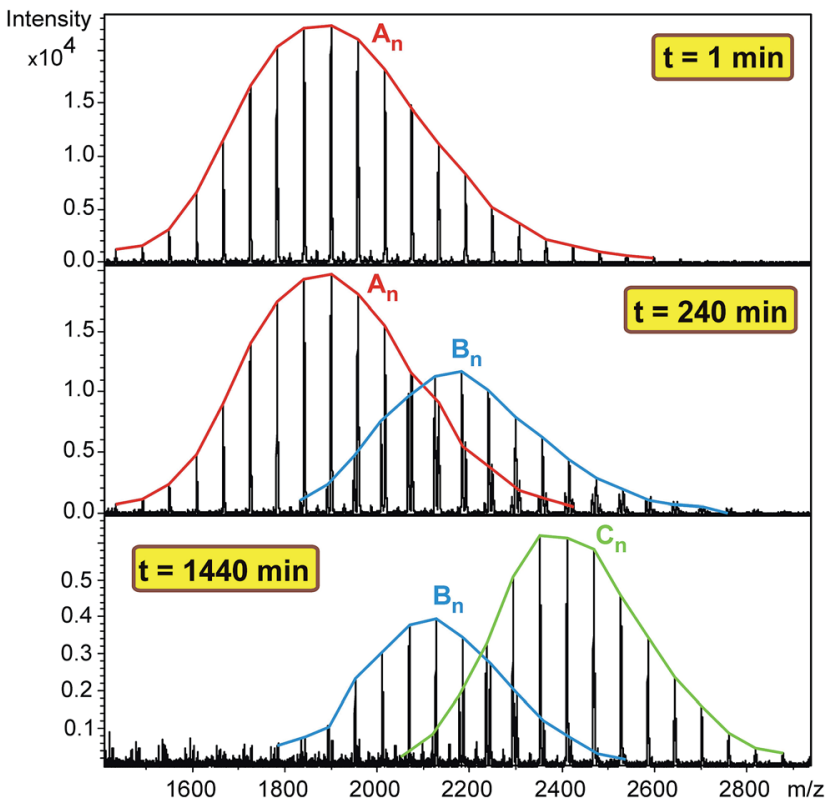

Fig. 1 MALDI-TOF MS spectra of the quenched reaction mixture obtained in the reaction of PPG with MDI at 1, 240 and 1440 minutes. Experimental conditions: $[\mathrm{MDI}]_{\circ}=0.32 \mathrm{M},[\mathrm{PPG}]_{\circ}=0.01 \mathrm{M}$ and $T=80$ ${ }^{\circ} \mathrm{C}$.
As seen in Fig. 1 the MALDI-TOF MS spectrum obtained at 1 min reaction time shows the presence of the starting PPG only. The $m / z$ values of the members of series $\mathrm{A}_{n}$ cationized by sodium ions are in good agreement with those calculated for the PPG having two hydroxyl groups. For example, the measured and the calculated monoisotopic masses for the PPG with a number of propylene oxide (PO) units 32 are 1898.2 and 1898.3, respectively. As the reaction time is increased, series $\mathrm{B}_{n}$ begin to appear. The $m / z$ value for $\mathrm{B}_{n}, e . g$. with a number of PO units 32 is 2180.5 while the calculated value for this composition is 2180.4. Further increase in the reaction time resulted in the disappearance of the starting diol and the dominant presence of series $\mathrm{C}_{n}$ in which both hydroxyl groups have been reacted. In addition, by comparing the MALDI-TOF MS spectra shown in Fig. 1, it can also be concluded that the molecular weight distributions shifts stepwise by a mass of $282 \mathrm{Da}$ from series $\mathrm{A}_{n}$ to $\mathrm{C}_{n}$. Note that the nominal mass of an MDI plus a methanol unit is $282 \mathrm{Da}$. PPG with two diisocyanate end-groups have the same mass as those having an allophanate end-group, i.e., those of which obtained by the reaction of hydroxyl group diisocyanate followed by addition of a second diisocyanate unit to the urethane bond formed. Thus, in order to confirm the formation of PPG with two diisocyanate end-groups and to rule out the formation of allophanate bonds under the experimental conditions employed, additional experiments using electrospray ionization tandem mass spectrometry (ESI-MS/MS) were performed. For these ESIMS/MS experiments several adduct ions, preferentially doubly charged sodiated adduct ions $\left([\mathrm{M}+2 \mathrm{Na}]^{2+}\right)$ containing two MDI units were subjected to collision induced dissociation (CID) to obtain structural information on PPG with two diisocyanate groups. A representative ESI-MS/MS spectrum obtained by fragmentation of precursor ions with $\mathrm{m} / \mathrm{z} 1213.7$ is shown in Fig. 2.

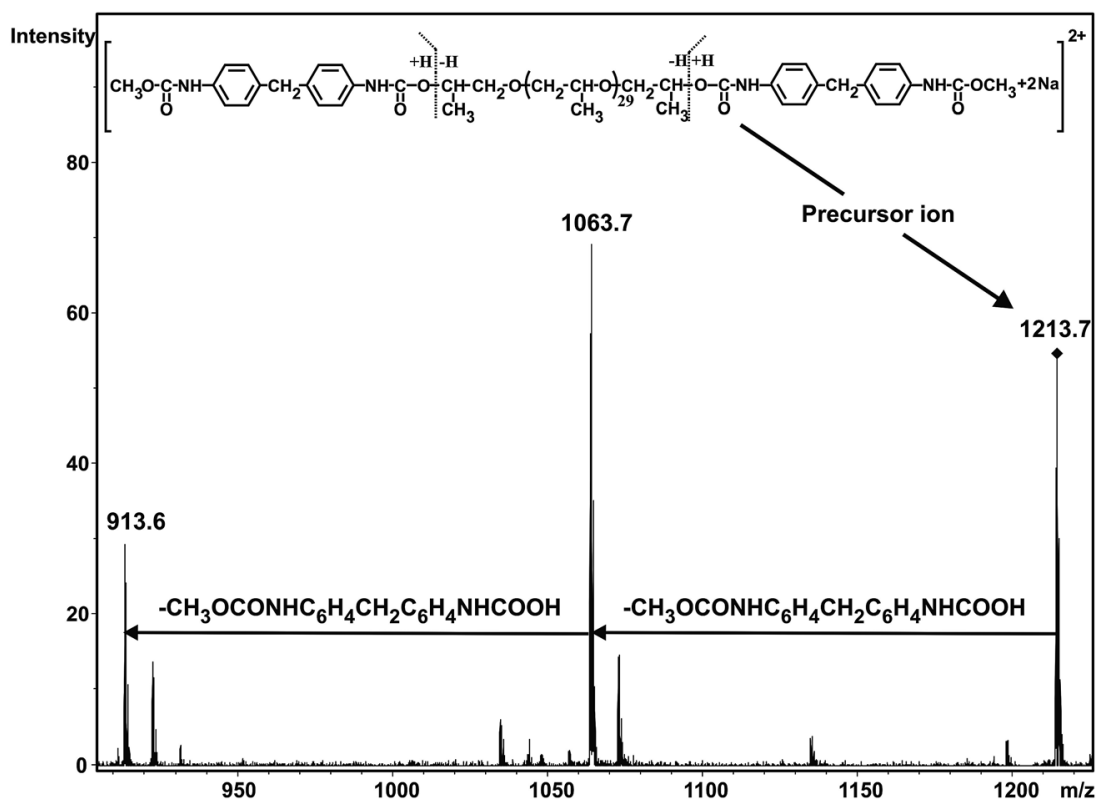

Fig. $2 \mathrm{ESI}-\mathrm{MS} / \mathrm{MS}$ spectrum of the $\left[\mathrm{PPG}+2 \mathrm{MDI}+2 \mathrm{CH}_{3} \mathrm{OH}+2 \mathrm{Na}\right]^{2+}$ adduct ion with a number of repeat units of $31(\mathrm{~m} / \mathrm{z} 1213.7)$. The ESI-MS/MS spectrum was recorded at collision energy of $178 \mathrm{eV}$. 
Fig. 2 suggests that the main fragmentation pathway is the subsequent loss of two $\mathrm{CH}_{3} \mathrm{OCONHC}_{6} \mathrm{H}_{4} \mathrm{CH}_{2} \mathrm{C}_{6} \mathrm{H}_{4} \mathrm{NHCOOH}$ units from the precursor ion. The fact that these two units are eliminated is a clear indication of the presence of the diisocyanate end-groups, i.e., both hydroxyl groups of PPG have been reacted with diisocyanate.

Monitoring the MDI-polymer diols reaction in time and determining the composition of the reaction mixtures one can obtain information on the reactivity of the MDI towards the hydroxyl groups of polymer diols. The relative MALDI-TOF MS response factors were estimated experimentally using the procedure presented in the Experimental, and $f_{\mathrm{A}} / f_{\mathrm{B}} \approx f_{\mathrm{A}} / f_{\mathrm{C}} \approx 1$ was found. Similar observations were found for the other polymer diols (i.e., PTHF and PCLD) investigated. Changes in the molar fractions for series $\mathrm{A}_{n}, \mathrm{~B}_{n}$ and $\mathrm{C}_{n}$ with the reaction time are depicted in Fig. 3.

Variations of the molar fractions for $\mathrm{A}_{n}, \mathrm{~B}_{n}$, and $\mathrm{C}_{n}$ in Fig. 3 reveal characteristic features of typical consecutive reactions. It should be noted, that no additional series can be detected with significant intensities. Fig. 4 shows the partial MALDI-TOF MS spectrum of the reaction products obtained in the reaction of MDI with PCLD.

As it turns out from Fig. 4, all the reaction products of the MDI-PCLD reaction, i.e., series $\mathrm{A}_{n}, \mathrm{~B}_{n}$ and $\mathrm{C}_{n}$, are well-resolved. They can clearly be distinguished from one another, which allows us to depict the variation in the composition of the reaction mixture in time (Fig. 5).

The run of the $X_{\mathrm{i}}$ versus time traces is similar to those obtained in the MDI-PPG reaction. Similar kinetic curves were also obtained for the MDI-PTHF reaction, too (shown in the ESI as Fig. S1 $\dagger$ ).

In order to support the validity of the consecutive reactions presented in Scheme 1 and to determine the corresponding values of $k_{1}$ and $k_{2}$, eqn (10)-(12) were fitted to the experimental

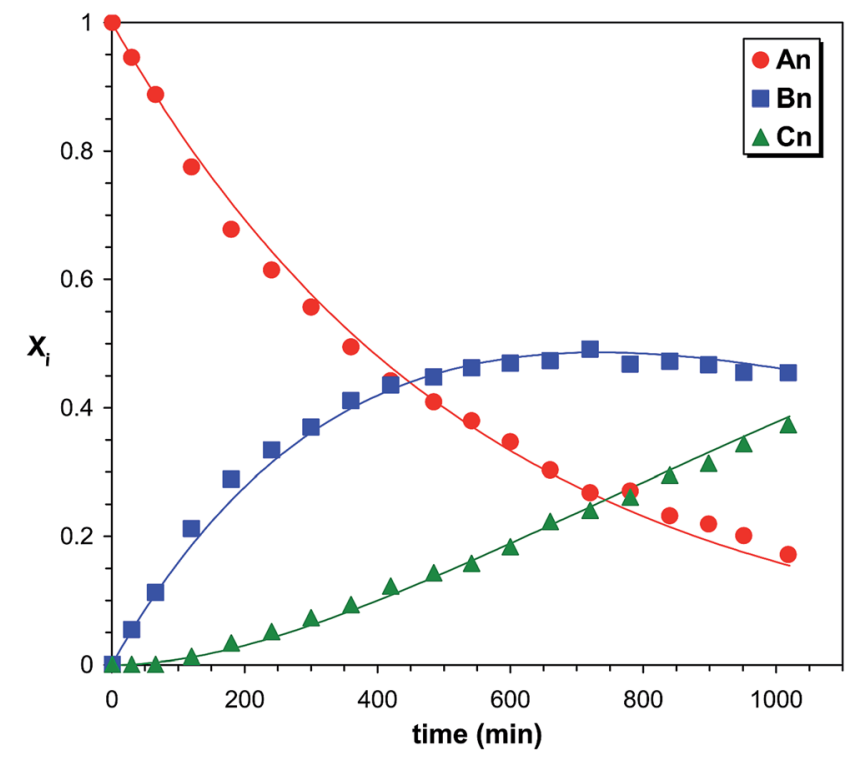

Fig. 3 Variations of the product distributions with time in the PPGMDI reaction determined by MALDI-TOF MS. The solid lines represent the fitted curves calculated by eqn (10)-(12). Experimental conditions: see Fig. 1 caption.

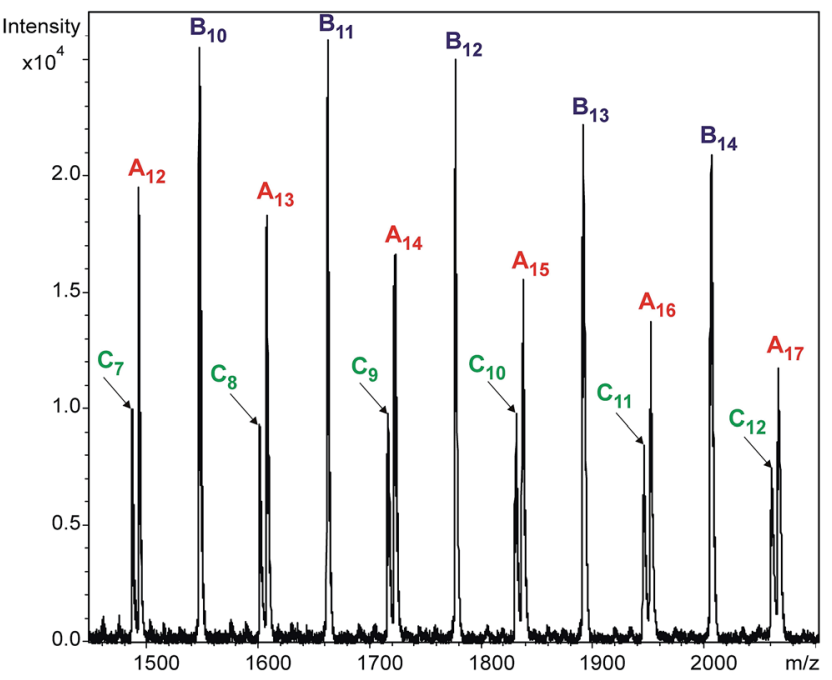

Fig. 4 MALDI-TOF MS spectrum of the quenched reaction mixture obtained in the reaction of PCLD with MDI at 40 minutes. The numbers in the subscript refer to the number of caprolactone units. Experimental conditions: $[\mathrm{MDI}]_{\circ}=0.32 \mathrm{M},[\mathrm{PCLD}]_{\circ}=0.01 \mathrm{M}$ and $T=80^{\circ} \mathrm{C}$.

molar fraction versus reaction time data. The solid lines in Fig. 3 and 5 show the results of such fits. As seen in Fig. 3 and 5 the fitted curves match reasonable well the experimental data. The corresponding rate constants $k_{1}$ and $k_{2}$ are compiled in Table 1 .

Data in Table 1 indicate that PCLD and PTHF have similar reactivities towards MDI, while PPG reacts by almost one order of magnitude slower. Similar reactivities of PCLD and PTHF are most probably due to the presence of primary hydroxyl groups and relatively long carbon-chain-ends in both polymers. In contrast, the low reactivity of PPG can preferentially be ascribed to the secondary hydroxyl end-groups in PPG. It is well-known

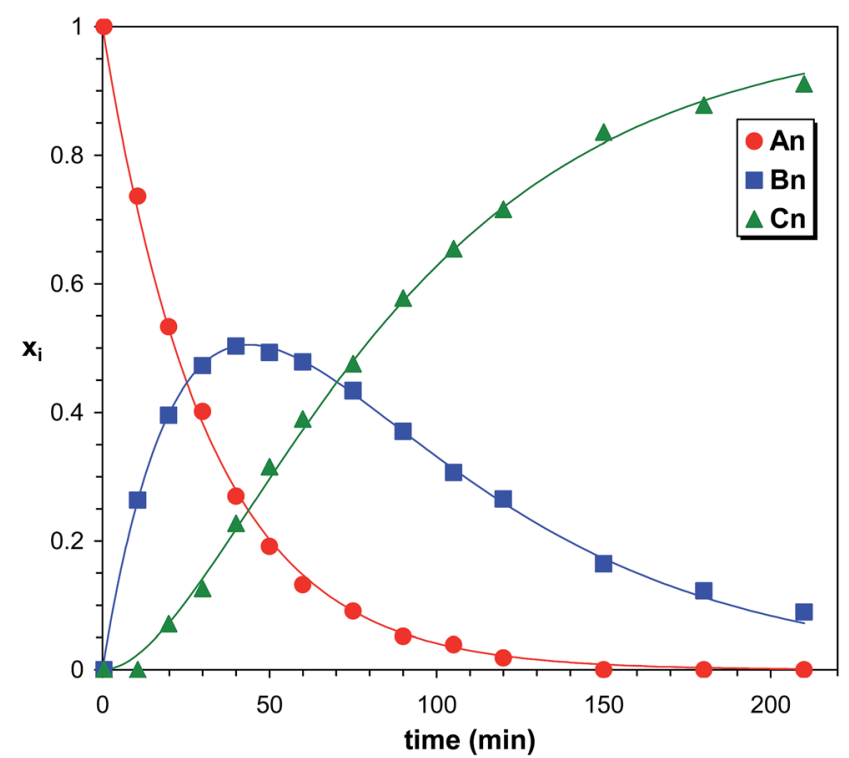

Fig. 5 Product distributions versus time in the PCLD-MDI reaction determined by MALDI-TOF MS. The solid lines represent the fitted curves calculated by eqn (10)-(12). Experimental conditions: see Fig. 4 caption. 
Table 1 The pseudo first-order rate constants for the reactions of MDI with polymer diols (PPG, PTHF and PCD) obtained by fitting the parameters of eqn (10)-(12) to the experimental curves. $k_{1}$ and $k_{2}$ are the rate constants for the reactions of MDI with the first and the second hydroxyl group (i.e., when the first one has been reacted), respectively. Experimental conditions: $[\mathrm{MDI}]_{\mathrm{O}}=0.32 \mathrm{M}$, [polymer diol]。 $=0.01 \mathrm{M}, T=80^{\circ} \mathrm{C}$ solvent: toluene

\begin{tabular}{lcl}
\hline Polymer diol & $k_{1} \times 10^{4}\left(\mathrm{~min}^{-1}\right)$ & $k_{2} \times 10^{4}\left(\mathrm{~min}^{-1}\right)$ \\
\hline PPG & $4.6 \pm 0.1$ & $4.9 \pm 0.2$ \\
PTHF & $51 \pm 1$ & $59 \pm 1$ \\
PCLD & $80 \pm 1$ & $78 \pm 2$
\end{tabular}

that secondary hydroxyl groups are less reactive than the primary ones. ${ }^{19,20}$ Thus, to confirm the presence of secondary hydroxyl end-groups in the PPG sample, the primary and the secondary hydroxyl group contents were determined according to a previously reported method. ${ }^{41,42}$ It was found that PPG contained $\sim 10 \%$ primary and $\sim 90 \%$ secondary hydroxyl endgroups. It is also evident from the data of Table 1 that the values of $k_{1}$ are very close to those of $k_{2}$, i.e., $k_{2} / k_{1}$ values are 1.06 , 1.16 and 0.98 for PPG, PTHF and PCLD, respectively. This finding indicates that after either of the two hydroxyl groups has been reacted the reactivity of the remaining is not altered significantly, i.e., the absence of substitution effect. Another way to obtain data for the extent of substitution effect is to plot $X_{\mathrm{B}_{n}}$ as a function of $X_{\mathrm{A}_{n}}$. This kind evaluation of kinetic data allows us not only to determine the values of $k_{2} / k_{1}$ but deviation from the statistically expected case, i.e., presence or absence of substitution effect, can easily be visualized over a wide range of $X_{\mathrm{A}_{n}}-X_{\mathrm{B}_{n}}$ data. eqn (10)-(12) can easily be transformed into a time-independent relationship between $X_{\mathrm{A}_{n}}$ and $X_{\mathrm{B}_{n}}$ as shown by eqn (21) (the derivation of eqn (21) can be found in the ESI $\dagger$ ).

$$
X_{\mathrm{B}_{n}}=\frac{X_{\mathrm{A}_{n}}{ }^{\alpha}-X_{\mathrm{A}_{n}}}{1-\alpha}
$$

where $\alpha=k_{2} / 2 k_{1}$.

It follows from eqn (21) that the maximum value of $X_{\mathrm{B}_{n}}$ $\left(X_{\mathrm{B}_{n}, \max }\right)$ can be given by eqn (22) as:

$$
X_{\mathrm{B}_{n}, \max }=\frac{\alpha^{\frac{\alpha}{1-\alpha}}-\alpha^{\frac{1}{1-\alpha}}}{1-\alpha}
$$

It can also be deduced from eqn (22) that in the case of $\alpha=$ 0.5 , i.e., in the absence of substitution effect, the value of $X_{\mathrm{B}_{n}, \text { max }}$ is 0.5 .

In Fig. $6 X_{\mathrm{B}_{n}}$ as a function of $X_{\mathrm{A}_{n}}$ is presented for PPG, PTHF and PCLD.

As it turns out from Fig. 6, the experimental $X_{\mathrm{A}_{n}}$ versus $X_{\mathrm{B}_{n}}$ values for PPG, PTHF and PCLD are very close to those calculated by eqn (21) with $\alpha=0.5$, supporting the absence of any significant substitution effect in the MDI-polymer diol reactions.

\section{Reaction of polypropylene glycol glycerol triether (PPG_GL) with MDI}

Similarly to the MDI-polymer-diol systems, the polymer-triol PPG_GL reacts in stepwise with MDI during the formation of

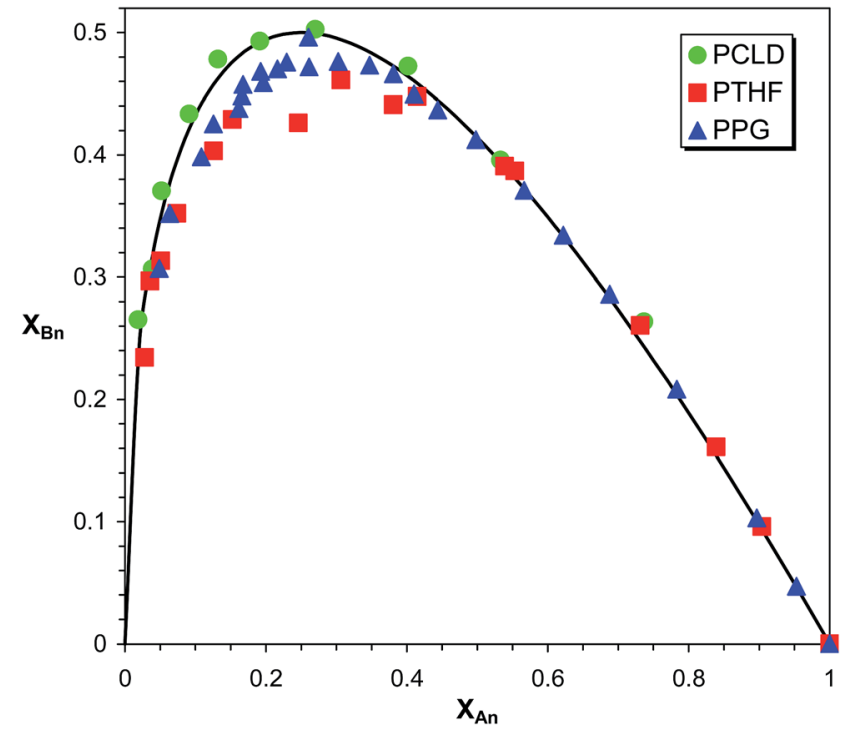

Fig. 6 Variation of $X_{\mathrm{B}_{n}}$ as a function of $X_{\mathrm{A}_{n}}$ for PPG, PTHF and PCLD. The solid line show the theoretical curve for the case of $\alpha=0.5$.

series $\mathrm{B}_{n}, \mathrm{C}_{n}$ and $\mathrm{D}_{n}$ as shown in Scheme 2. The formation of these series is evidenced by the MALDI-TOF MS spectra of the methanol-quenched reaction mixture recorded at different reaction times (Fig. 7).

As seen in Fig. 7 after a short reaction time only the starting PPG_GL is present. However, at elapsed reaction time, the formation of series $\mathrm{B}_{n}, \mathrm{C}_{n}$ and $\mathrm{D}_{n}$ can also be detected, while at very long reaction time the final product (series $\mathrm{D}_{n}$ ) appears with the highest intensity. Furthermore, the masses of series increase by $282 \mathrm{Da}$ from series $\mathrm{A}_{n}$ to $\mathrm{D}_{n}$, corresponding to the

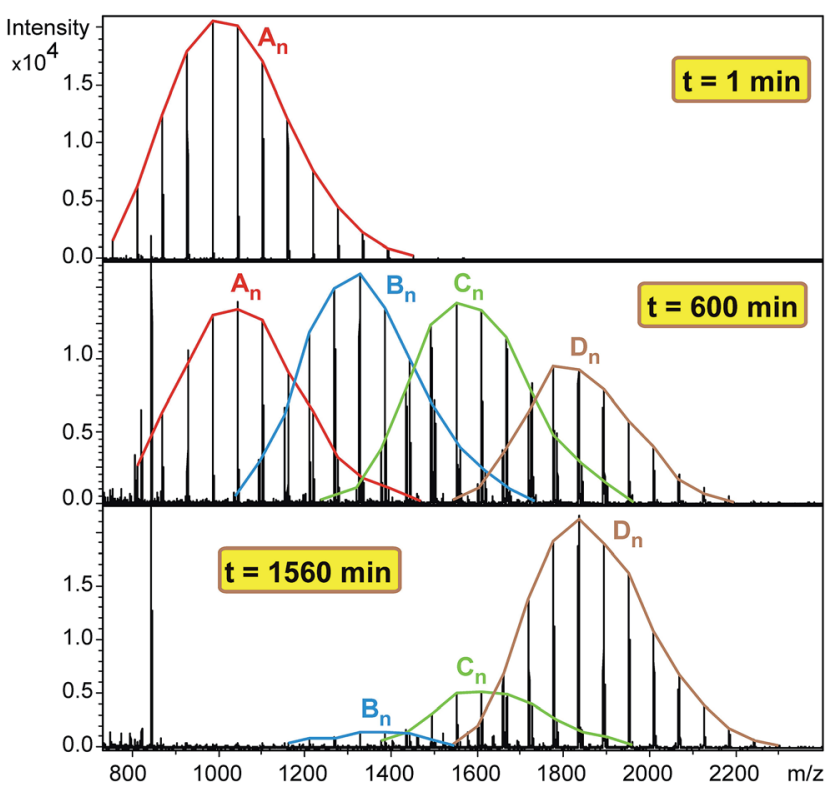

Fig. 7 MALDI-TOF MS spectra of the quenched reaction mixture obtained in the reaction of PPG_GL with MDI at 1, 600 and 1560 minutes. Experimental conditions: $[\mathrm{MDI}]_{\mathrm{O}}=0.32 \mathrm{M}$, $\left[\mathrm{PPG} \mathrm{GL}_{\mathrm{O}}=0.01\right.$ $\mathrm{M}$ and $T=80^{\circ} \mathrm{C}$. 
subsequent additions of MDI molecules to the hydroxyl groups of PPG_GL. The ESI-MS/MS spectrum (shown in the ESI as Fig. $\mathrm{S} 2 \dagger)$ on selected precursor ions from series $\mathrm{B}_{n}, \mathrm{C}_{n}$ and $\mathrm{D}_{n}$ show consecutive losses of $\mathrm{CH}_{3} \mathrm{OCONHC}_{6} \mathrm{H}_{4} \mathrm{CH}_{2} \mathrm{C}_{6} \mathrm{H}_{4} \mathrm{NHCOOH}$ units. This confirms that the reaction of MDI with all of the three hydroxyl groups of PPG_GL took place and no allophanates formed under the reaction conditions employed. The relative MALDI-TOF MS response factors for series $\mathrm{B}_{n}, \mathrm{C}_{n}$ and $\mathrm{D}_{n}$ were found to be $f_{\mathrm{A}} / f_{\mathrm{B}} \approx 1.3$ and $f_{\mathrm{A}} / f_{\mathrm{C}} \approx f_{\mathrm{A}} / f_{\mathrm{D}} \approx 2.1$, respectively. The fractional MALDI-TOF MS intensities were then converted into molar fractions using the corresponding relative MALDITOF MS response factors. Eqn (17)-(20) were fitted to the experimental molar fractions versus time data to determine the corresponding rate constants $k_{1}, k_{2}$ and $k_{3}$ (Fig. 8).

The determined values of $k_{1}, k_{2}$ and $k_{3}$ were $(4.7 \pm 0.1) \times$ $10^{-4} \min ^{-1},(8.5 \pm 0.2) \times 10^{-4} \mathrm{~min}^{-1}$ and $(8.6 \pm 0.2) \times 10^{-4}$ $\min ^{-1}$, respectively. Note that these rate constant values,

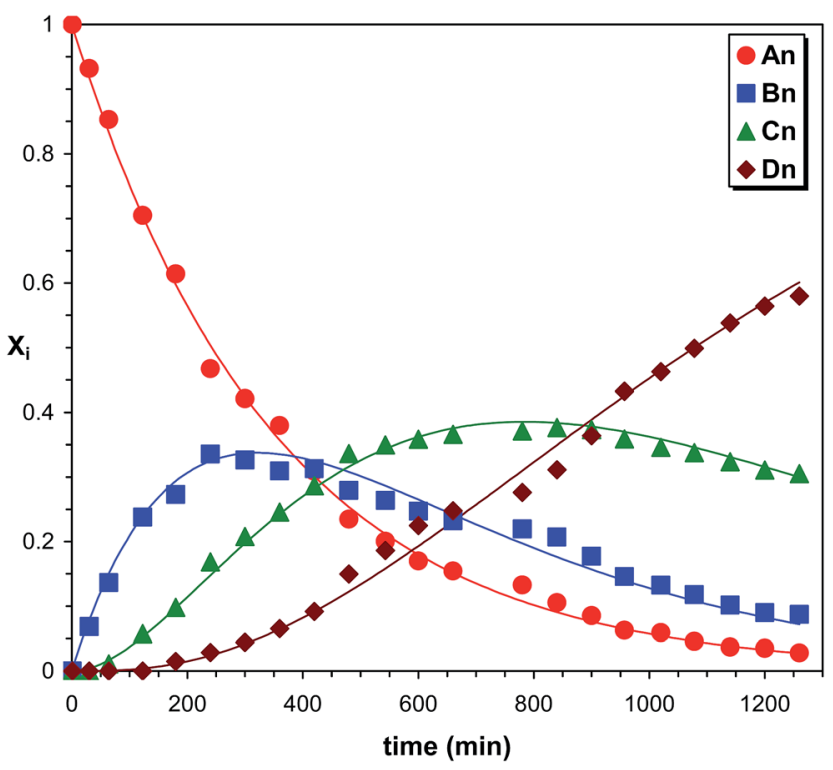

Fig. 8 Product distributions versus time in the PPG_GL-MDI reaction determined by MALDI-TOF MS. The solid lines represent the fitted curves calculated by eqn (17)-(20). Experimental conditions: see Fig. 7 caption. especially $k_{1}$, are very close to those of PPG (Table 1 ). Although the values of $k_{2}$ and $k_{3}$ are very similar, both are higher than $k_{1}$, indicating a positive substitution effect. This means that the reaction of the hydroxyl groups with MDI proceeds faster after the first one has been reacted.

Furthermore, using the $X_{\mathrm{B}}$ versus $X_{\mathrm{A}}$ data shown in Fig. 9a, the $\alpha$ parameter can be determined using eqn (21). In case of a trifunctional triol $\alpha=2 k_{2} / 3 k_{1}$ holds. Accordingly, using eqn (21) $\alpha=1.20 \pm 0.02$ was obtained.

In addition, the variation of the molar fraction of series $\mathrm{C}_{n}$ $\left(X_{\mathrm{C}_{n}}\right)$ with that of series $\mathrm{A}_{n}\left(X_{\mathrm{A}_{n}}\right)$ can be expressed as shown by eqn (23) (the derivation of eqn (23) can be found in the ESI $\dagger$ ).

$$
\begin{aligned}
X_{\mathrm{C}_{n}}= & \frac{\alpha}{(1-\alpha)(1-\alpha \beta)}\left(X_{\mathrm{A}_{n}}-X_{\mathrm{A}_{n}}{ }^{\alpha \beta}\right) \\
& +\frac{1}{(1-\alpha)(1-\alpha \beta)}\left(X_{\mathrm{A}_{n}}^{\alpha \beta}-X_{\mathrm{A}_{n}}{ }^{\alpha}\right)
\end{aligned}
$$

where $\alpha=2 k_{2} / 3 k_{1}$ and $\beta=k_{3} / 2 k_{2}$.

By fitting eqn (23) to the experimental $X_{\mathrm{C}_{n}}$ versus $X_{\mathrm{A}_{n}}$ data (see Fig. 9b) the values of $\alpha$ and $\beta$ can be determined for which $\alpha=$ $1.23 \pm 0.02$ and $\beta=0.51 \pm 0.01$ were obtained. It is noteworthy that very similar $\alpha$ values were deduced from both the $X_{\mathrm{B}_{n}}$ versus $X_{\mathrm{A}_{n}}$ and the $X_{\mathrm{C}_{n}}$ versus $X_{\mathrm{A}_{n}}$ plots. In the absence of substitution and/or any other effects the values of the rate constants $k_{1}, k_{2}$ and $k_{3}$ should be equals, yielding values of $2 / 3$ and 0.5 for $\alpha$ and $\beta$, respectively. In the case of "pure" statistical attachments of MDI units to the hydroxyl groups of PPG_GL the maximum value of both $X_{\mathrm{B}_{n}}$ and $X_{\mathrm{C}_{n}}$ are expected to be $4 / 9$. The latter value can be obtained by derivation of eqn (22) and (23) and inserting $\alpha=2 / 3$ and $\beta=0.5$ ). However, as it is evident from Fig. 9 the maxima of both $X_{\mathrm{B}_{n}}$ and $X_{\mathrm{C}_{n}}$ are considerable lower than $4 / 9$ and thus $\alpha$ is higher than $2 / 3$ suggesting a positive substitution effect. The ${ }^{1} \mathrm{H}-\mathrm{NMR}$-analysis of the PPG_GL sample treated with $\left(\mathrm{CF}_{3} \mathrm{CO}\right)_{2} \mathrm{O}$ (similarly to that presented for PPG) revealed the dominant presence of secondary hydroxyl groups (>98\%) with less than $2 \%$ primary hydroxyl group content. Hence, deviations from the theoretical ones can not be explained by any significant presence of hydroxyl groups with different reactivities. Considering the molecular weight of PPG_GL $\left(M_{\mathrm{n}}=1000 \mathrm{~g}\right.$ $\mathrm{mol}^{-1}$ ) one can estimate that one "arm" PPG_GL is composed of ca. 5-6 PO units on the average, thus representing relatively
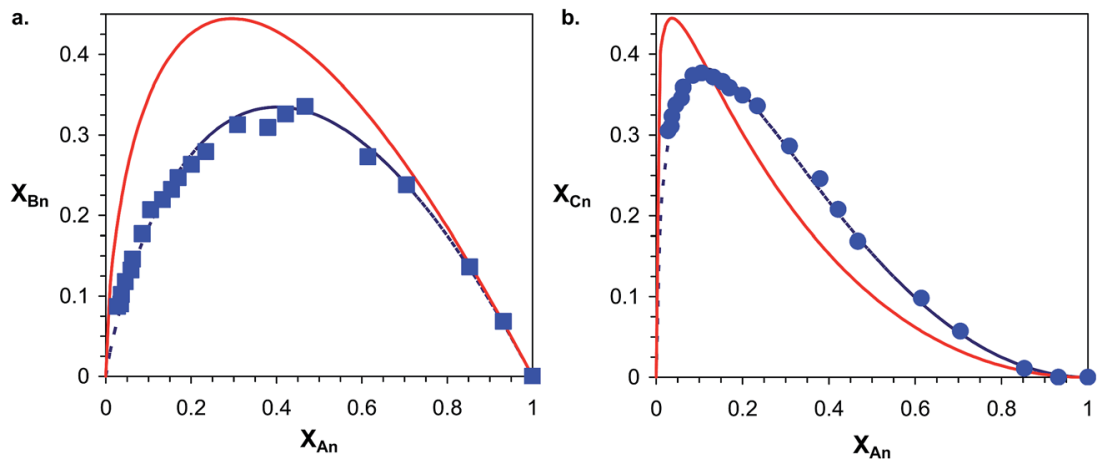

Fig. 9 Variation of $X_{B_{n}}(a)$ and $X_{C_{n}}$ (b) as a function of $X_{A_{n}}$ for PPG_GL. The solid lines show the theoretical curves for the cases of $\alpha=2 / 3$ (a) and $\alpha=2 / 3$ and $\beta=0.5$, while the dotted lines represent the best fitting with $\alpha=1.20$ (a) and $\alpha=1.23$ and $\beta=0.51$. 
short "arms". Based on this argument, we may conclude that the observed positive substitution effect may be due to a facile interaction between the urethane and the unreacted hydroxyl end-groups. However, it cannot be excluded that the substitution effect observed for the MDI-PPG_GL system may come from the uncertainties associated with the determinations of the relative MALDI-TOF MS response factors and/or the occurrence of "unobserved" side reactions.

\section{Conclusion}

The progress of the reactions of three polymer polyols including PPG, PTHF and PCLD with MDI was monitored by MALDI-TOF MS. The resulting MALDI-TOF MS intensities obtained in the methanol-quenched reaction mixture at different reactions times allowed us to construct the fractional intensities for the corresponding polymer series appeared in the MALDI-TOF MS spectra. The fractional intensities were then converted into molar fractions using the relative MALDI-TOF MS response factors. From the molar fractions versus time data the corresponding pseudo firstorder rate constants were determined and the reactivities of the polymer-diols were found to decrease in the order of PCLD $>$ PTHF $\gg$ PPG. The low reactivity of PPG compared to those of PCLD and PTHF were ascribed to the presence of secondary hydroxyl groups in PPG which were confirmed by ${ }^{1} \mathrm{H}-\mathrm{NMR}$ analysis. Based on the results of kinetic experiment it was concluded that the urethane end-group formed in the reaction of $\mathrm{OH}$ group with MDI does not alter significantly the reactivity of the unreacted hydroxyl group in the case of these polymer-diols. However, the reaction of PPG_GL with MDI represented a more complex kinetic situation as, besides the starting one, three additional polymer series corresponding to the PPG_GL reacted with one, two and three MDI units were formed. The relative MALDI-TOF MS response factors for the different polymer series were also estimated and these response factors were used further to obtain the molar fractions for the different polymer series from the corresponding fractional intensities. Using the molar fractions versus time data and a consecutive reaction model consisting of three reaction steps, the corresponding pseudo-first order rate constants $\left(k_{1}, k_{2}\right.$ and $\left.k_{3}\right)$ were determined. According to the results, the rate constant $k_{1}$ determined for PPG_GL was very close to that obtained for PPG indicating that the number of "arms" and the molecular weight had no significant effect on the reactivities of the terminal hydroxyl groups. Moreover, the reaction of the MDI with the second hydroxyl group of PPG_GL (i.e. after one of the three has been reacted) proceeds faster than with the first one, indicating a positive substitution effect. This effect may be attributed to a facile interaction between the urethane group formed and the yet unreacted hydroxyl end-groups owing to the presence of the relatively short "arms" in PPG_GL. Moreover, we can not exclude that the substitution effect observed for the MDI-PPG_GL system may arise from uncertainties of the determinations of the relative MALDI-TOF MS response factors and/or the presence of "unobserved" side reactions.
In addition, it has been shown that the time-independent $X_{\mathrm{A}_{n}}-X_{\mathrm{B}_{n}}$ plot for the MDI-polymer-diol and the $X_{\mathrm{A}_{n}}-X_{\mathrm{B}_{n}}$ and the $X_{\mathrm{A}_{n}}-X_{\mathrm{C}_{n}}$ plots for the MDI-polymer-triol systems yielded reliable results for the relative reactivities and the presence or absence of any substitution effects.

It has been also demonstrated that MALDI-TOF MS offers unique way for studying such a complex polymer systems as each of the species with different masses can easily be identified and quantitative information can also be gained providing that the corresponding response factors are successfully determined.

\section{Acknowledgements}

The authors acknowledge financial support for this work by grant No. K-101850 from OTKA (National Scientific Research Fund, Hungary). This work was also supported by Hungarian National Talent Program (NTP-EFÖ-P-15, T. Nagy).

\section{References}

1 A. A. Caraculacu and S. Soceri, Prog. Polym. Sci., 2001, 26, 799.

2 P. Król, Prog. Mater. Sci., 2007, 52, 915.

3 R. J. Zdrahala and I. J. Zdrahala, J. Biomater. Appl., 1999, 14, 67.

4 A. Lendlein and R. Langer, Science, 2002, 296, 1673.

5 B. K. Kim, S. Y. Lee and M. Xu, Polymer, 1996, 37, 5781.

6 K. Czifrák, J. Karger-Kocsis, L. Daróczi, M. Zsuga and S. Kéki, Macromol. Chem. Phys., 2014, 215, 1896.

7 F. H. Brock, J. Chem. Soc., 1961, 65, 1638.

8 J. Burkus and C. F. Eckert, J. Am. Chem. Soc., 1957, 80, 5948.

9 J. W. Baker and J. Gaun, J. Chem. Soc., 1949, 19.

10 E. Dyer, H. A. Taylor, S. J. Mason and J. Samson, J. Chem. Soc., 1949, 71, 4106.

11 G. Anzuino, A. Pirro, O. Rossi and L. Polo Friz, J. Polym. Sci., Polym. Chem. Ed., 1975, 13, 1657.

12 G. Raspoet, M. T. Nguyen, M. McGarraghy and A. F. Hegarty, J. Org. Chem., 1998, 63, 6878.

13 A. A. Caraculacu, I. Agherghinei, M. Gapar and C. Prisacariu, J. Chem. Soc., Perkin Trans. 2, 1990, 8, 1343.

14 B. Grepinet, F. Pla, P. H. Hobbes, P. H. Swaels and T. H. Monge, J. Appl. Polym. Sci., 2000, 75, 705.

15 P. Król and J. Wojturska, J. Appl. Polym. Sci., 2003, 88, 327. 16 X. Y. Huang, W. Yu and C. S. P. Sung, Macromolecules, 1990, 23, 391.

17 X. D. Sun and C. S. P. Sung, Macromolecules, 1996, 29, 3198. 18 Z. H. Gao, J. Y. Gu and X. D. Bai, Pigm. Resin Technol., 2007, 36, 90.

19 M. A. Zaverkina, V. P. Lodygina, V. V. Komratova, E. V. Stovbun and E. R. Badamshina, Polym. Sci., Ser. A, 2006, 48, 382.

20 T. Nagy, B. Antal, K. Czifrak, I. Papp, J. Karger-Kocsis, M. Zsuga and S. Keki, J. Appl. Polym. Sci., 2015, 132, 42127.

21 A. Kaushik and P. Singh, Int. J. Polym. Mater., 2008, 57, 815. 22 T. N. M. T. Ismail, K. D. P. Palam, Z. B. A. Bakar, H. S. Soi, Y. S. Kian, H. A. Hassan, C. Schiffman, A. Sendijarevic, 
V. Sendijarevic and I. Sendijarevic, J. Appl. Polym. Sci., 2016, 133, 42955.

23 C. A. Cateto, M. F. Barreiro, A. E. Rodrigues and M. N. Belgacem, React. Funct. Polym., 2011, 71, 863.

24 M. Ionescu, Z. S. Petrovic and X. Wan, J. Polym. Environ., 2010, 18, 1.

25 P. K. Maji and A. K. Bhowmick, J. Polym. Sci., Part A: Polym. Chem., 2009, 47, 731.

26 U. Sebenik and M. Krajnc, Int. J. Adhes. Adhes., 2007, 27, 527.

27 M. A. Semsarzadeh and A. H. Navarchian, J. Polym. Eng., 2003, 23(4), 225.

28 C. Pavier and A. Gandini, Eur. Polym. J., 2000, 36, 1653.

29 M. J. Elwell, A. J. Ryan, H. J. M. Grünbauer and H. C. Van Lieshout, Macromolecules, 1996, 29, 2960.

30 M. Möller and H. U. Moritz, J. Appl. Polym. Sci., 2006, 101, 4090.

31 R. Ghoreishi and G. J. Suppes, RSC Adv., 2015, 5, 68361.

32 A. Eceiza, K. de la Caba, V. Gascón, M. A. Corcuera and I. Mondragon, Eur. Polym. J., 2001, 37, 1685.

33 R. Ghoreishi, Y. Zhao and G. J. Suppes, J. Appl. Polym. Sci., 2014, 131, 40388.
34 P. Król and B. Pilch-Pitera, Polymer, 2003, 44, 5075.

35 A. Eceiza, K. de la Caba, G. Kortaberria, N. Gabilondo, C. Marieta, M. A. Corcuera and I. Mondragon, Eur. Polym. J., 2005, 41, 3051.

36 B. Grepinet, F. Pla, P. H. Hobbes, P. H. Swaels and T. H. Monge, J. Appl. Polym. Sci., 2001, 81, 3149.

37 H. Kothandaraman and A. Sultan Nasar, J. Appl. Polym. Sci., 1993, 50, 1611.

38 K. Tanaka, H. Waki, Y. Ido, S. Akita and T. Yoshida, Rapid Commun. Mass Spectrom., 1988, 2, 151.

39 S. F. Wong, C. K. Meng and J. B. Fenn, J. Phys. Chem., 1988, 92, 546.

40 K. M. Kadish, X. Mu and J. E. Anderson, Pure Appl. Chem., 1989, 16, 1823.

41 M. Bednarek, P. Kubisa and S. Penczek, Macromol. Chem. Phys., 1989, 15, 49.

42 D. Chakraborty, A. Rodriguez and E. Y. X. Chen, Macromolecules, 2003, 36, 5470.

43 D. Marquardt, SIAM J. Appl. Math., 1963, 11, 431. 